which the force between glass plates (optically flat to within $300 \mathrm{~A}$.) was determined over the range $0 \cdot 7-1 \mu$, indicate that the force at a separation of $1 \mu$ is $1.2( \pm 0.4) \times 10^{-3}$ dyne per $\mathrm{cm}^{2}$, and the index of $d$ is $3 \cdot 9 \pm 0 \cdot 3$. Hence, the value of $B$ in equation (2) is $1.2($ 土 0.4$) \times 10^{-19} \mathrm{erg} \mathrm{cm}$.

The theory of the dispersion forces at large distances is therefore confirmed, in full agreement with the results of Derjaguin and Abricossova. In preliminary observations we also observed large forces similar to those reported by Overbeek and Sparnaay; these disappeared on application of a high-frequency discharge or when water-vapour was admitted to the apparatus. These observations add support to the suggestion made by Derjaguin and Abricossova that such forces originate from mosaic electrostatic charges.

Details of this work will be published elsewhere.
A. P. Prosser
J. A. Kitchener

Department of Chemistry,

Imperial College of Science and Technology,

$$
\text { London, S.W.7. }
$$

Oct. 10.

${ }^{1}$ de Boer, J. H., Trans. Farad. Soc., 32, 21 (1936).

${ }^{2}$ Casimir, H. B. G., and Polder, D., Phys. Rev., '73, 360 (1948).

${ }^{3}$ Lifshitz, E., Exp. Theor. Phys. USSR, 29, 94 (1955). Soviet Physics JETP, 2, 73 (transl.) (1956).

${ }^{4}$ Derjaguin, B., and Abricossova, I., J. Exp. Theor. Phys. USS R, 21 945 (1951); C.R. Acad. Sci. UU.S.S.R., 90, 1055 (1953); Disc. Farad. Soc., 18, 33, 183 (1954).

'Overbeek, J. Th. G., and Sparnaay, M. J., Proc. K. Akad. Wetensch., B54, 387 (1951); J. Colloid Sci., 7, 343 (1952); Dise. Farad. Soc. 18, 12 (1954)

\section{A General Expression for Intermolecular Potentials}

A systematic project for the evaluation of virial coefficients (second, third and fourth) by automatic computation has been in operation on the EDSAC (Cambridge) : tabulated results and mathematical procedures will be given elsewhere. This investigation, however, gave rise to the consideration of a related problem to which the following interesting solution has been found.

In the estimations of intermolecular potentials of spherical molecules to fit observed virial coefficients and related properties, there are a few well-known investigation $^{1}$ in which different expressions with two or three adjustable parameters have been tested and advocated. However, it appears desirable, in general, and particularly with respect to the present automatic calculations, to introduce a new expression. which has an unlimited number of adjustable constants such as the coefficients of a power series. Such an expression should enable one to make further progress than by the trial of miscellaneous expressions: the agreement for any expression of tabulated functions with two or three parameter's must presumably be limited and would be weakened by further improvement and extension of experimental data. This attempt at a systematic procedure seemed to be specially desirable for the most useful organization of the numerical results in the above investigation.

A few different types of expression were examined and the following was selected:

$U(r)=\frac{4 \varepsilon}{\left(r^{2}+B^{2}\right)^{3}} \sum_{i=0} C_{2 i}\left\{r^{2 i} \exp A\left(1-r^{2}\right)-1\right\}$
The intermolecular distance $r$ is to be measured so that the potential $U(1)$ at unity is zero. The constants $A$ and $B^{2}$ are to be assigned values about 4 and $1 / 10$, but the constants $C_{2} i$ are to be adjusted to fit experimental or fundamental theoretical data, and their free choice makes the expression dependent on a complete system of functions. The 4 and $1 / 10$ choice has the surprising property that with $C_{0}=1$ and other $C_{2 i}=0$, the function is practically indistinguishable from the Lennard-Jones (12-6) potential,

$$
V(r)=4 \varepsilon\left(\frac{1}{r^{12}}-\frac{1}{r^{6}}\right)
$$

This degree of agreement had not been thought possible and is very gratifying, since the use of $U$ provides thus a first term which is probably nearly as good as possible for a two-parameter expression while still permitting unlimited further adjustment.

T'able 1

\begin{tabular}{|c|c|c|}
\hline \multirow{2}{*}{$r$} & \multicolumn{2}{|c|}{$U(r) / 4 \varepsilon$} \\
\hline 0.80 & $\begin{array}{c}\text { Potential (1) with } \\
C_{0}=1.0 ; C_{2} i=0(i>0)\end{array}$ & $\begin{array}{c}(12-6) \\
\text { potential }\end{array}$ \\
\hline 0.90 & 7.948 & 10.737 \\
0.94 & 1.511 & 1.659 \\
0.98 & 0.623 & 0.652 \\
1.00 & 0.144 & 0.146 \\
1.02 & 0.000 & 0.000 \\
1.06 & -0.101 & -0.099 \\
1.10 & -0.213 & -0.208 \\
1.12 (min.) & -0.253 & -0.246 \\
1.16 & -0.257 & -0.250 \\
1.2 & -0.248 & -0.242 \\
1.4 & -0.227 & -0.223 \\
1.6 & -0.112 & -0.115 \\
2.0 & -0.053 & -0.056 \\
2.0 & -0.015 & -0.015 \\
\hline
\end{tabular}

It does not appear likely that any powerful systematic way for evaluating $C_{0}, C_{2}, C_{4}$ from experimental data will be found. Their estimation will have to depend on solid-state and viscosity data as well as on the virial coefficients. However, some procedure of successive adjustment of the $C_{2 i}$ appears to offer for the first time a systematic way of sum. marizing the implications of the experimental data. It may also be hoped that this systematization of approach might be of some use in the selection of most effective experimental measurements.

In the work just performed on the EDSAC, the second, third and fourth virial coefficients for the basic potential with $\left(C_{2}=C_{4}=\ldots=0\right)$ and for two others with $\left(C_{6}=C_{8}=\ldots=0\right)$ have been calculated. Linear combinations of these can then be examined by means of the tabulated results to estimate the best functions to fit experimental data. It may be of interest to note that the calculation depended neither on the analytical form of the potential nor on numerical integration. It was based on an expansion of $\exp (-U / k T)-1$ in terms of functions of the type $\exp \left(-a r^{2}\right)$. Practically all the organization and arithmetic of the evaluations were performed by the machine.

I. ShavitT

S. F. Boys

Theoretical Chemistry Department, University of Cambridge.

Oct. 9 .

"Lirschfelder, J. O., Curtiss, C. F., and Bird, R. B., "Molecular Theory of Gases and Liquids", see chapter 1 (1954). 